\title{
RESEARCH
}

Open Access

\section{Chloroethylating anticancer drug-induced mutagenesis and its repair in Escherichia coli}

Yoko Yamada', Shinji Watanabe ${ }^{2}$, Keinosuke Okamoto ${ }^{1,2,4}$, Sakae Arimoto ${ }^{1,2}$, Eizo Takahashi ${ }^{1,3,4}$, Kazuo Negishi $^{3}$ and Tomoe Negishi ${ }^{1,3^{*}}$ (D)

\begin{abstract}
Background: Chloroethylnitrosourea (CENU) derivatives, such as nimustine (ACNU) and carmustine (BCNU), are employed in brain tumor chemotherapy due to their ability to cross the blood-brain barrier. They are thought to suppress tumor development through DNA chloroethylation, followed by the formation of interstrand cross-links (ICLs) that efficiently block replication and transcription. However, the alkylation of DNA and ICLs may trigger genotoxicity, leading to tumor formation as a side effect of the chemotherapeutic treatment. Although the involvement of $O^{6}$-alkylguanine-DNA alkyltransferase (AGT) in repairing chloroethylated guanine $\left(O^{6}\right.$ chloroethylguanine) has been reported, the exact lesion responsible for the genotoxicity and the pathway responsible for repairing it remains unclear.
\end{abstract}

Results: We examined the mutations induced by ACNU and BCNU using a series of Escherichia coli strains, CC101 to CC111, in which reverse mutations due to each episome from $F^{\prime} 101$ to $F^{\prime} 106$ and frameshift mutations due to each episome from F'107 to $F^{\prime} 111$ could be detected. The mutant frequency increased in E. coli CC102, which can detect a GC to AT mutation. To determine the pathway responsible for repairing the CENU-induced lesions, we compared the frequency of mutations induced by CENU in the wild-type strain to those in the ada, ogt (AGTdeficient) strain, uvrA (nucleotide excision repair (NER)-deficient) strain, mismatch repair (MMR)-deficient strains, and recA (recombination deficient) strain of E. coli CC102. The frequencies of mutations induced by ACNU and BCNU increased in the ada, ogt strain, demonstrating that $O^{6}$-chloroethylguanines were formed, and that a portion was repaired by AGT.

Mutation induced by ACNU in NER-deficient strain showed a similar profile to that in AGT-deficient strain, suggesting that an NER and AGT play at the similar efficacy to protect $E$. coli from mutation induced by ACNU. $O^{6}$ Chloroethylguanine is reported to form ICLs if it is not repaired. We examined the survival rates and the frequencies of mutations induced by ACNU and BCNU in the uvrA strain, the recA strain, as well as a double-deficient strain of CC102. The mutation profile of the double-deficient strain was similar to that of the NER-deficient strain, suggesting that an NER protects E. coli from mutations but not recombination. In addition, cell death was more pronounced in the uvrA, recA double-deficient strain than in the single-deficient strains.

(Continued on next page)

\footnotetext{
* Correspondence: tomoeisaka@gmail.com

${ }^{1}$ Graduate School of Medicine, Dentistry, and Pharmaceutical Sciences,

Okayama University, Tsushima-naka, Kita-ku, Okayama 700-8530, Japan

${ }^{3}$ Nihon Pharmaceutical University, Ina, Kita-Adachi-Gun, Saitama 362-0806,

Japan

Full list of author information is available at the end of the article
}

(c) The Author(s). 2019 Open Access This article is distributed under the terms of the Creative Commons Attribution 4.0 International License (http://creativecommons.org/licenses/by/4.0/), which permits unrestricted use, distribution, and reproduction in any medium, provided you give appropriate credit to the original author(s) and the source, provide a link to the Creative Commons license, and indicate if changes were made. The Creative Commons Public Domain Dedication waiver (http://creativecommons.org/publicdomain/zero/1.0/) applies to the data made available in this article, unless otherwise stated. 
(Continued from previous page)

Conclusion: These results suggest that the toxic lesions induced by CENU were repaired additively or synergistically by NER and recombination. In other words, lesions, such as ICLs, appear to be repaired by NER and recombination independently.

Keywords: Chloroethylnitrosourea, Nimustine (ACNU), Carmustine (BCNU), DNA repair, Recombination, Interstrand cross-link (ICL), Escherichia coli,

\section{Background}

Although many kinds of alkylating agents have been traditionally used as anti-cancer therapeutic agents, numerous studies have reported that alkylating agents can cause tumors due to genotoxicity, and that the DNA repair systems are strongly involved in expression of anti-carcinogenic and genotoxic functions [1-4]. In many cases, alkylation such as methylation, ethylation, or chloroethylation, at the $O^{6}$ site of guanine causes DNA lesions that induce cytotoxicity and genotoxicity. Chloroethylnitrosoureas (CENUs), such as nimustine (ACNU) and carmustine (BCNU), are typical chloroethylating agents that are employed in tumor chemotherapy for treating several kinds of tumors, including lymphomas, melanomas, small cell lung cancer, Hodgkin disease, and cerebromas $[5,6]$. The blood-brain barrier is a major obstacle in therapies for brain tumors [7], and since CENUs can cross the blood-brain barrier, they are useful and important as chemotherapeutic agents against brain tumors [8]. CENUs are thought to cause chloroethylation at several DNA sites, and among them, $\mathrm{O}^{6}$-chloroethylguanine is considered to be the most influential lesion in causing cytotoxicity and genotoxicity [4]. Alkylation of the $O^{6}$ site of guanine is well-known to cause a base substitution from GC to AT [9]. $O^{6}$-Chloroethylguanine is unstable and immediately transforms into $\mathrm{N} 1-\mathrm{O}^{6}$-ethenoguanine via circularization of its molecule as an intermediate to form an interstrand cross-link (ICL) between guanine and cytosine [10]. ICLs strongly inhibit DNA replication [11], and these ICL lesions are considered to be responsible for the cytotoxic effects of CENUs $[4,5]$. However, ICLs might trigger not only cytotoxicity, but also genotoxicity, leading to tumor formation as a side effect of the chemotherapeutic treatment $[5,11,12]$. The involvement of $O^{6}$-alkylguanine-DNA alkyltransferase (AGT) in the repair of chloroethylating guanine has been widely reported [6, 13-15]. Recently, the mechanism of ICL-repair as well as the cytotoxic effects of ICLs has been slightly clarified [11]. Previously Wiencke and Wiemels reported that BCNU was weakly mutagenic in Ames test using Salmonella typhymurium hisG46 and TA1535 that detect the base substitution from $\mathrm{G}$ to A [16]. However, the relationship between the genotoxic lesions and mutagenesis remains unclear, and its repair pathway remains to be fully elucidated. In this study, we examined the mutations induced by $\mathrm{ACNU}$ and $\mathrm{BCNU}$ in E. coli strains CC101 to CC111, which can be used to detect reverse mutations due to each episome from F'101 to F'106 and frameshift mutations due to each episome from F'107 to F'111 [17, 18]. The mutantion frequency increased only in the E. coli CC102 strain, in which a GC to AT mutation was detected. In an exception, BCNU was mutagenic in $E$. coli CC104 strain only at high dose. Frameshift mutations were not detected in assays using strains $\mathrm{CC} 107$ to $\mathrm{CC} 111$. To determine the pathway responsible for repairing the CENU-induced lesions, we examined the frequencies of mutations induced by CENUs in the ada, ogt (AGT)-deficient strain, uvrA (NER)-deficient strain, mismatch repair (MMR)-deficient strain, $r e c A$ (recombination)-deficient strain, and $u v r A$ and $r e c A$ double-mutant strain of CC102. The frequencies of mutations induced by ACNU and BCNU were elevated in the ada, ogt strain, indicating that $\mathrm{O}^{6}$-chloroethylguanine was formed, then repaired in part by AGT. The pathway for ICL repair is complicated, and it might be related to recombination. A significant decrease in survival was observed in the double-deficient $u \nu r A, \operatorname{rec} A$ strain, while the mutant frequency was similar to that of the NER single-deficient strain, suggesting that in E. coli, NER and AGT prevent GC to AT mutations, and that NER and recombination independently prevent cytotoxic effects.

\section{Methods}

Materials

The ACNU (nimustine hydrochloride; 1-[(4-amino-2-meth yl-5-pyrimidinyl)-.methyl]-3-(2-chloroethyl)-3-nitrosourea hydrochloride; CAS 55661-38-6) was purchased from Wako Pure Chemicals Co. (Osaka, Japan), and the BCNU (carmustine; 1,3-bis(2-chloroethyl)-1-nitrosourea; CAS 154 -93-8) and temozolomide (TMZ; 3,4-dihydro-3-methy1-4-oxoimidazo-[5,1-d] [1,2,3,5]tetrazine-8-carboxamide; CA S 85622-93-1) were purchased from Tokyo Chemical Industry Co., Ltd. (Tokyo, Japan). The structures of these compounds are shown in Fig. 1.

\section{Bacterial strains}

Escherichia coli strains, each containing an F' episome from CC101 to CC106, were used to detect base 
$N$-Chloroethyl $N$-nitrosoureas

Nimustine (ACNU)<smiles>Cc1ncc(CNC(=O)N2CCCCC2)c(N)n1</smiles>

Carmustine (BCNU)

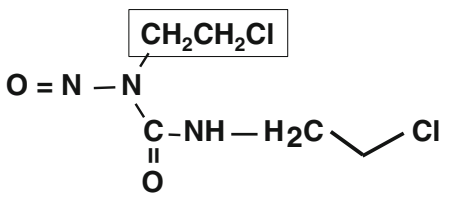

Temozolomide (TMZ)<smiles>Cn1nnc2c(C(N)=O)ncn2c1=O</smiles>

Fig. 1 The structures of the mutagens used in this study

substitutions [17], and other E. coli strains, each containing an $\mathrm{F}^{\prime}$ episome from $\mathrm{CC} 107$ to $\mathrm{CC} 111$, were used to detect frameshift mutations [18]. The repair-deficient mutant strains with the CC102 episome used in this study are summarized in Table 1 . All E. coli strains used in the mutagenesis experiments were derived from strain KA796 (ara, thi and $\Delta p r o-l a c$ ) [19]. The wild-type strain (NR10832), the MMR-deficient derivatives (NR12896 (mutS201::Tn5), NR11102 (mutL211::Tn5), and NR12897 (mutH471::Tn5)), the NER-deficient derivative (NR12999 (uvrA277::Tn10)), and the recombination-deficient derivative (NR11312 (recA56, srl::Tn10)) have all been described by Negishi et al. [19]. The AGT-deficient mutant KT01121 (ada-10::Tn10, ogt::cat) has been reported by Taira et al. [20]. To lack the AGT activity completely, we use the double mutant in which both inducible gene (ada)-product (Ada) and constitutive gene (ogt)-product (Ogt) is deficient. Another NER-deficient strain, ZA2102 (uvrA6, malE::Tn5), was kindly gifted from Prof. T. Ohta
(Tokyo University of Pharmacy and Life Sciences, Tokyo) [21]. A double mutant with uvrA and recA, SW102 (uvrA6, malE::Tn5, recA56, srl::Tn10), was constructed in this study using NR11312 as a host strain and ZA2102 as a donor strain, according to methods described previously $[20,22]$. We confirmed that this strain was sensitive to ultraviolet (UV) irradiation and ENU due to a NER deficiency and a recombination deficiency, respectively.

\section{Bacterial mutation assay}

The lac allele of CC102 reverts to $\mathrm{lac}^{+}$exclusively through a GC to AT transition [17]. We previously used this lac reversion system to detect the mutagenicity of alkylating agents in wild-type and mismatch-repair-deficient strains [20, 23, 24]. For mutagenesis, $0.1 \mathrm{ml}$ of overnight cultures of each strain were incubated for $1 \mathrm{~h}$ at $37^{\circ} \mathrm{C}$ with $0.5 \mathrm{ml}$ of $0.1 \mathrm{M}$ sodium phosphate buffer ( $\mathrm{pH} 7.4)$ and $0.1 \mathrm{ml}$ of mutagen solution dissolved in DMSO or water. We assayed in triplicate. Next, $0.1 \mathrm{ml}$ of the treated cultures

Table 1 E. coli KA796 mutants used in this study and their spontaneous mutant frequencies

\begin{tabular}{llll}
\hline Strain $^{a}$ & Relevant genotype & $\begin{array}{l}\text { Spontaneous mutant frequency } \\
\left(\times 10^{-7}\right)\end{array}$ & Ref. $^{b}$ \\
\hline NR12999 & KA796, uvrA277:::Tn10 & $0.24 \pm 0.16$ & {$[18]$} \\
KT01121 & KA796, ada-10::Tn10, ogt::cat & $0.78 \pm 0.27$ & {$[19]$} \\
NR12896 & KA796, mutS201::Tn5 & $59.6 \pm 11.4$ & {$[18]$} \\
NR11102 & KA796, mutL211::Tn5 & $22.5 \pm 9.63$ & {$[18]$} \\
NR12897 & KA796, mutH471:::Tn5 & $43.0 \pm 16.5$ & {$[18]$} \\
NR11312 & KA796, recA56, srl::Tn10 & $0.16 \pm 0.1$ & {$[18]$} \\
ZA2102 & uvrA6, malE::Tn5 & $<0.1$ & {$[20]$} \\
SW102 & uvrA6, malE::Tn5, recA56, srl::Tn10 & $0.1 \pm 0.5$ & This study \\
\hline
\end{tabular}

a: All mutants contain CC102 F'episome

$b$ : All spontaneous mutant frequencies shown in this table were measured in this study 
were spread onto minimal lactose plates to determine the number of revertants, and adequately diluted cultures were also spread onto minimal glucose plates to determine the total viable cell numbers. The doses of mutagens used in these assays were scarcely toxic. In almost all cases, the survival rates were greater than $80 \%$, and in only a few cases did the survival rate decrease to approximately 60 to $70 \%$ at the highest dose used. The mutation frequencies were calculated by dividing the number of $\mathrm{lac}^{+}$revertants by the number of total viable cells. In the experiment using double mutant ( $u v r A$ and $\operatorname{rec} A$ ), we performed the assay at lower dose of test compounds because $\mathrm{ACNU}$ and $\mathrm{BCNU}$ were more toxic in double mutant than in each single mutant. All experiments were independently repeated two or three times. Typical results are shown in Figs. 2, 3 and 4. Statistical analysis was performed using the Student's $t$ test.

\section{Results}

Mutation spectrum induced by ACNU and BCNU

Although the study of mutations induced by CENUs has mainly been performed in mammalian cells, in this study, we used an E. coli reversion assay to investigate the lesions resulted in CENU-induced mutations. First, we determined which base substitution or frameshift was induced by treatment with $\mathrm{ACNU}$ or BCNU using a series of E. coli strains, from CC101 to CC111. As shown in Table 2, the mutation frequency in CC102 increased significantly only after treatment with either compound, that is, a GC to AT transition occurred, whereas no other base substitution was detected, except for a GC to $\mathrm{TA}$ transversion resulting from $\mathrm{BCNU}$ treatment. No frameshift mutation was observed. In CC104, mutation was significantly detected with a higher dose of $\mathrm{BCNU}$ (5.19 $\pm 0.52 \times 10^{-5}$ at $1 \mathrm{mM}, p<0.01$; compared to 0.45 \pm 0.03 at $0 \mathrm{mM}$ ), suggesting that $\mathrm{BCNU}$ might also induce a GC to TA transversion. In the Ames test, ACNU and BCNU were mutagenic in TA100, $717 \pm 194$ revertants/ $\mu \mathrm{mol}$ and $1042 \pm 122$ revertants/ $\mu \mathrm{mol}$ respectively, and ACNU was weakly, mutagenic in TA98 $(92 \pm 8.2$ revertants $/ \mu \mathrm{mol})$. These results suggest that $A C N U$ and BCNU induce a base substitution, that is, a GC to AT transition, and $\mathrm{ACNU}$ also induce frameshift mutation, that is, $+1(\mathrm{C} / \mathrm{G})$.

\section{Effects of DNA repair deficiencies on the frequency of mutations induced by ACNU and BCNU}

To determine which DNA lesions were induced by $\mathrm{ACNU}$ and $\mathrm{BCNU}$, we examined the repair systems that can prevent the mutations induced by $\mathrm{ACNU}$ and BCNU using DNA repair-deficient mutants derived from CC102. An ada, ogt strain (KT01121) was used to examine the involvement of $O^{6}$-chloroethylated guanine in the mutation, a NER-deficient strain (uvrA; MR12999) was used to examine not only chloroethyl but also more bulky adducts on the DNA, and a recombination-deficient strain (recA; NR11312) was used to examine ICLs in the DNA. As shown in Fig. 2a, BCNU was more mutagenic in the $a d a, o g t$ strain than in the wild-type strain, and the mutation frequency did not differ significantly between the
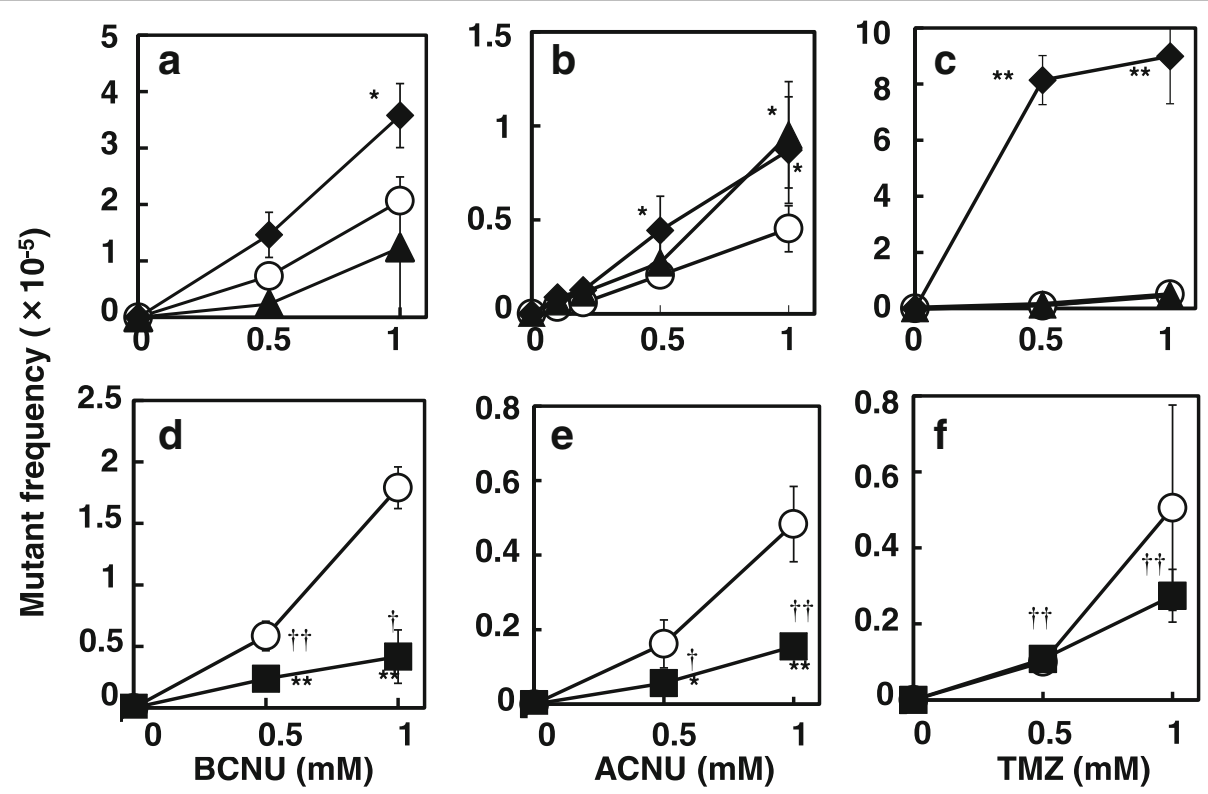

Fig. 2 Mutant frequencies induced by BCNU $(\mathbf{a}, \mathbf{d}), \operatorname{ACNU}(\mathbf{b}, \mathbf{e})$, and TMZ (c, $\mathbf{f})$ in the wild-type (open circles), KT01121 (closed diamonds), NR12999 (closed triangles), and NR11312 (closed squares) strains. Statistical analysis was performed using the Student's $t$ test. ${ }^{* *} p<0.01$ and ${ }^{*} p<0.05$ when compared to the mutation frequency of the wild-type strain. $+\uparrow p<0.01$ and $+p<0.05$ when compared to the mutant frequency of control without treatment 

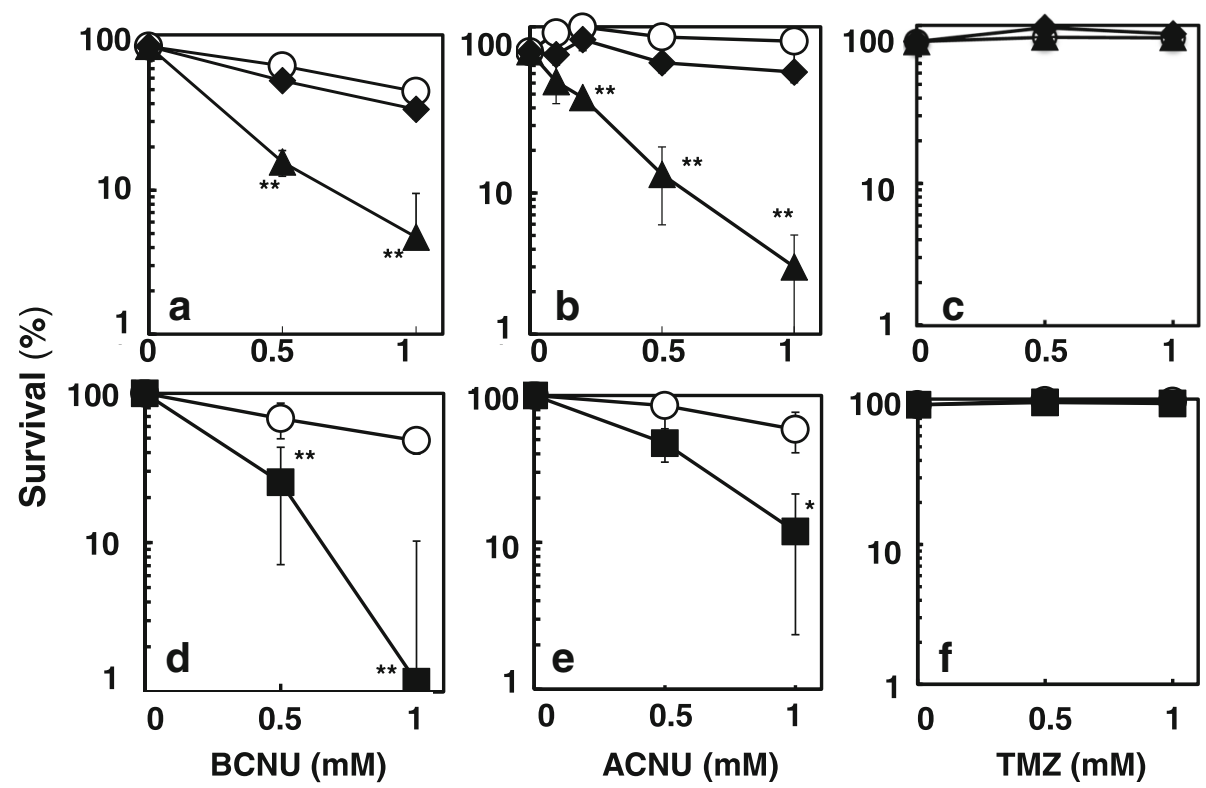

Fig. 3 Cytotoxicity induced by BCNU (a, d), ACNU (b, e), and TMZ (c, f) in the wild-type (open circles), KT01121 (closed diamonds), NR12999 (closed triangles), and NR11312 (closed squares) strains. Statistical analysis was performed using the Student's $t$ test. ${ }^{*} p<0.01$ and ${ }^{*} p<0.05$ when compared to the mutant frequency of the wild-type strain
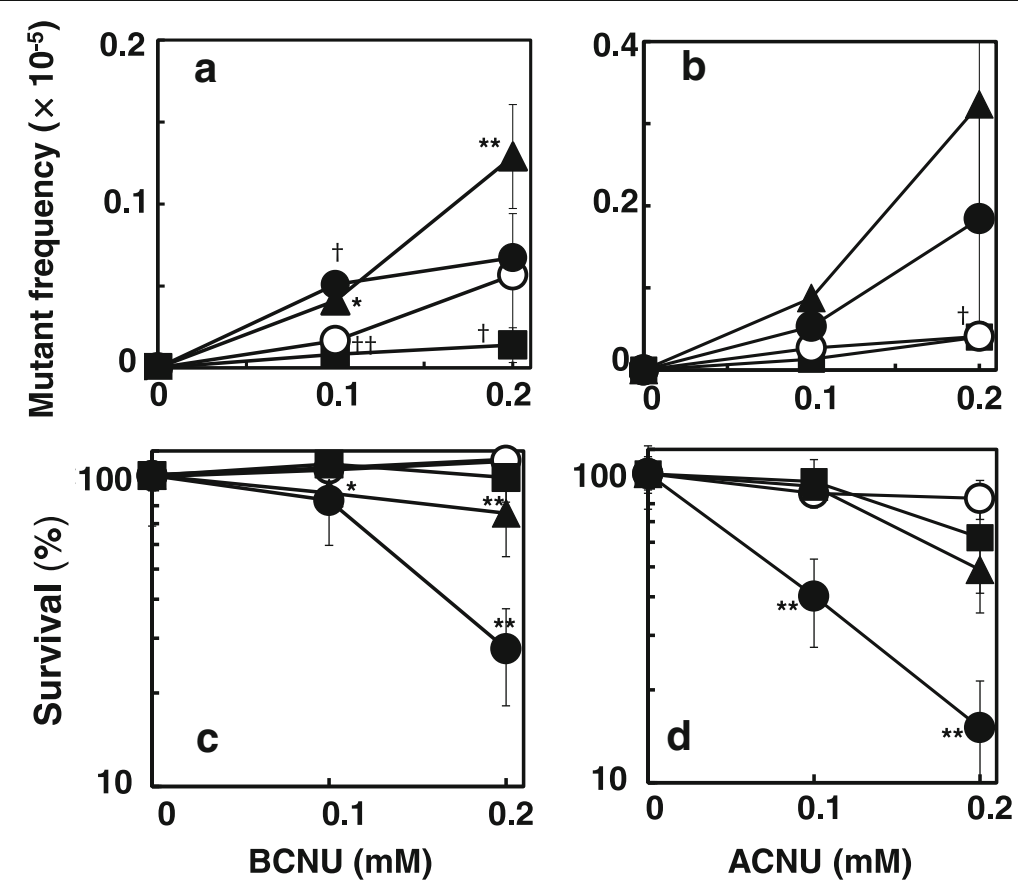

Fig. 4 Frequency of mutations and cytotoxicity induced by BCNU (a, c) and ACNU (b, d) in the wild-type (open circles), NR12999 (closed triangles), NR11312 (closed squares), and SW102 (closed circles) strains. Statistical analysis was performed using the Student's $t$ test. ${ }^{* *} p<0.01$ and ${ }^{*} p<0.05$ when compared to the mutation frequency of the wild-type strain. $+t p<0.01$ and $+p<0.05$ when compared to the mutant frequency of control without treatment 
Table 2 Mutant frequencies induced by ACNU and BCNU in E. coli series from CC101 to CC111

\begin{tabular}{|c|c|c|c|c|c|c|}
\hline \multirow[t]{2}{*}{ Strain } & \multicolumn{3}{|l|}{$\mathrm{ACNU}$} & \multicolumn{3}{|l|}{$\mathrm{BCNU}$} \\
\hline & dose (mM) & $\mathrm{MF}\left(\times 10^{-7}\right)$ & survival (\%) & dose (mM) & $\mathrm{MF}\left(\times 10^{-7}\right)$ & survival (\%), \\
\hline CC101 & 0 & $0.24 \pm 0.42$ & 100 & 0 & $0.24 \pm 0.21$ & 100 \\
\hline (AT to CG) & 1 & $<0.21$ & $101.6 \pm 6.5$ & 0.5 & $0.72 \pm 0.06$ & $49.5 \pm 4.2$ \\
\hline CC102 & 0 & $0.42 \pm 0.43$ & 100 & 0 & $0.31 \pm 0.02$ & 100 \\
\hline (GC to AT) & 1 & $33.0 \pm 5.74^{* *}$ & $102.1 \pm 14.4$ & 0.5 & $79.63 \pm 23.6^{* *}$ & $48.3 \pm 10.5$ \\
\hline CC103 & 0 & $<0.17$ & 100 & 0 & $<0.11$ & 100 \\
\hline (GC to CG) & 1 & $<0.18$ & $94.1 \pm 11.1$ & 0.5 & $<0.21$ & $55.4 \pm 3.9$ \\
\hline CC104 & 0 & $0.31 \pm 0.27$ & 100 & 0 & $0.45 \pm 0.03$ & 100 \\
\hline (GC to TA) & 1 & $0.62 \pm 0.55$ & $100.5 \pm 12.8$ & 0.5 & $1.75 \pm 1.85$ & $60.2 \pm 13.3$ \\
\hline CC105 & 0 & $0.64 \pm 0.04$ & 100 & 0 & $0.31 \pm 0.53$ & 100 \\
\hline (AT to TA) & 1 & $<0.12$ & $151.0 \pm 15.7$ & 0.5 & $0.40 \pm 0.35$ & $63.6 \pm 9.5$ \\
\hline CC106 & 0 & $<0.16$ & 100 & 0 & $<0.16$ & 100 \\
\hline (AT to GC) & 1 & $<0.13$ & $108.4 \pm 13.6$ & 0.5 & $<0.22$ & $57.6 \pm 18.1$ \\
\hline CC107 & 0 & $10.56 \pm 1.86$ & 100 & 0 & $6.90 \pm 0.88$ & 100 \\
\hline$(+1(\mathrm{G} / \mathrm{C}))$ & 1 & $8.01 \pm 1.60$ & $107.6 \pm 12.6$ & 0.5 & $8.61 \pm 1.54$ & $65.8 \pm 11.6$ \\
\hline CC108 & 0 & $4.58 \pm 1.17$ & 100 & 0 & $3.39 \pm 1.04$ & 100 \\
\hline$(+1(\mathrm{G} / \mathrm{C}))$ & 1 & $2.67 \pm 0.70$ & $111.9 \pm 9.0$ & 0.5 & $2.83 \pm 2.18$ & $87.1 \pm 10.7$ \\
\hline CC109 & 0 & $58.37 \pm 7.21$ & 100 & 0 & $43.88 \pm 3.17$ & 100 \\
\hline$(+2(C G / G C))$ & 1 & $57.60 \pm 12.7$ & $109.2 \pm 8.1$ & 0.5 & $42.28 \pm 13.38$ & $91.4 \pm 37.0$ \\
\hline CC110 & 0 & $0.27 \pm 0.46$ & 100 & 0 & $0.12 \pm 0.20$ & 100 \\
\hline$(+1(A / T))$ & 1 & $0.67 \pm 0.30$ & $141.2 \pm 14.2$ & 0.5 & $0.36 \pm 0.32$ & $75.3 \pm 13.5$ \\
\hline CC111 & 0 & $3.47 \pm 0.18$ & 100 & 0 & $6.85 \pm 1.49$ & 100 \\
\hline$(-2(A / T))$ & 1 & $6.01 \pm 2.21$ & $67.4 \pm 3.8$ & 0.5 & $4.23 \pm 0.76$ & $90.6 \pm 24.3$ \\
\hline
\end{tabular}

**; $p<0.01$, significant increase from the corresponding control

uvrA strain and the wild-type strain. In contrast, ACNU was only slightly more mutagenic in the $a d a$, ogt strain than in the wild-type strain, and the mutagenicity in the ada, ogt strain was similar to that in the uvrA strain (Fig. 2b). In addition, the mutagenicity of TMZ, a typical methylating anticancer drug, was markedly elevated in the $a d a$, ogt strain (Fig. 2c). From these results, it was considered that the chloroethylated guanine at the $O^{6}$ position of the DNA was partly repaired by AGT, as has been previously reported [4, 6]; however, AGT might be less efficient in repairing damage from chloroethylation than damage from methylation. In addition, ACNU might cause other DNA damage that is repaired by NER.

Chloroethylated $O^{6}$-guanine sequentially forms ICLs via the circularization of ethenoguanine. It is thought that ICLs are repaired through recombination accompanied by NER. We examined the effects of recombination deficiency on the mutations induced by ACNU and BCNU using recA strain NR11312 (Fig. 2d and e) and the double-mutant $u v r A$, recA strain SW102 (Fig. 4a and $\mathrm{b})$. Both compounds were very weakly but significantly mutagenic in NR11312, and the frequency of mutations decreased when compared to the wild-type strain. Recombination deficiency did not affect the mutagenicity and toxicity of TMZ (Fig. 2f). In $u v r A, \operatorname{rec} A$ strain, the frequency of mutations induced by $\mathrm{ACNU}$ and BCNU appeared to be similar or slightly higher than that in the wild-type strain, and similar to that in the recA strain (Fig. 4a and b).

In summary, the mutation induced by $\mathrm{ACNU}$ and BCNU appears to be protected by AGT and NER whereas the efficacy of NER for BCNU-induced mutation appears to be lesser than that for ACNU. Recombination might assist in mutagenesis induced by $\mathrm{ACNU}$ and BCNU.

\section{Influences of DNA repair pathways on the} cytotoxicity induced by $\mathrm{ACNU}$ and $\mathrm{BCNU}$

ACNU and BCNU induce ICLs through $O^{6}$-chloroethylguanine. The ICLs are believed to be the cytotoxic lesions and are proposed to be repaired by the combined action of NER and recombination [11]. Therefore we examined the cytotoxicity of ACNU, BCNU and TMZ using NER-deficient (uvrA), recombination-deficient (recA) E. coli. ACNU and BCNU caused strong cytotoxicity in the NER-deficient strain and recA strain (Fig. 3a, b, d and e), but TMZ was not 
toxic in any of the strains at the doses tested (Fig. $3 \mathrm{c}$ and $\mathrm{f}$ ). These results show that NER and recombination remove ACNU- and BCNU-induced cytotoxic lesions. To examine the relationship between NER and recombination in the repair of the ACNU- and BCNU-induced cytotoxic lesions using $u v r A$, recA double mutant. The cytotoxic effects of $\mathrm{ACNU}$ and $\mathrm{BCNU}$ were much stronger in the $u v r A, \operatorname{rec} A$ strain than in the recA or $u v r A$ single-mutant strains (Fig. 4c and $d$ ). These results suggest that the lesions induced by CENUs might be repaired by NER and recombination independently.

\section{Effects of mismatch repair deficiencies on the frequency of mutations induced by ACNU and BCNU}

We also examined whether MMR is involved in the repair of DNA lesions induced by CENUs, because it is well-known that MMR pathway involves in the processing alkylated guanine lesions by repair or cytotoxic effects [23-26]. Our results demonstrated that MMR was not responsible for the repair of chloroethylated lesions (Table 3). Thus, chloroethylated guanine does not appear to be a target for MMR.

\section{Discussion}

Chloroethylnitrosoureas (CENUs), such as ACNU and $\mathrm{BCNU}$, are typical chloroethylating agents that are employed in tumor chemotherapy, especially for brain tumors due to their ability to pass through the blood-brain barrier [7]. CENUs cause the formation of $O^{6}$-chloroethylguanine, followed by the formation of an ICL via $N 1-O^{6}$-ethenoguanine, and this ICL lesion blocks replication, leading to the cytotoxic effects of the CENUs [3-6]. However, these lesions are considered to be a double-edged sword, because they can also be genotoxic and mutagenic, and can lead to tumor formation. Unfortunately, the genotoxic mechanism of CENUs remains unclear. In this study, we examined the mutagenic effects induced by CENUs and the systems that repair the mutagenic DNA damage using an E. coli reversion assay system. This system can detect sequence-specific mutations easily. Previously, Tashima et al. reported that recA-lacking $E$. coli was very sensitive to $\mathrm{BCNU}$, and that the lesions induced by $\mathrm{BCNU}$ are likely repaired by post-replication repair, that is, homologous recombination [27]; however, although they observed the cytotoxic effects of $\mathrm{BCNU}$, they did not examine the mutations induced by BCNU. In our study, we first investigated the mutation spectrum caused by ACNU and BCNU using E. coli strains CC101 to CC111. The results showed that both chemicals induced GC to AT transition mutations in the CC102 strain, suggesting that chloroethylation occurred at the $O^{6}$ position of guanine, because it has been reported that $O^{6}$-chloroethylguanine induces $\mathrm{GC}$ to $\mathrm{AT}$ transitions via mispairing (reviewed in 3). These results are corroborated by previous reports that the toxicity of alkylating agents increased in cells lacking AGT or $\mathrm{O}^{6}$-methylguanine-DNA methyltransferase (MGMT) [2-4]. These results are in agreement with our data showing mutagenicity in S. typhymurium TA100. The therapeutic efficiency of ACNU for tumors is dependent on the MGMT expression status in tumor cells [28]. ACNU-induced sister chromatid exchanges were efficiently protected in HeLa cells transduced with human MGMT [29]. Preuss et al. observed that the protective effect of MGMT against cytotoxicity was specific to certain agents, that is, MGMT showed stronger protection against the cytotoxic effects of ACNU than those of BCNU in HeLa cells [14]. In addition, Becker et al. reported that skin tumors induced by topically applied ACNU were protected from cytotoxicity when human MGMT was expressed in the mice skin [30]. We observed a significant increase in the frequency of mutations induced by $\mathrm{BCNU}$ and $\mathrm{ACNU}$ in $a d a$, ogt E. coli; in the case of ACNU, the mutant frequency was similar to that in the $u v r A$ strain, while the frequency of mutations induced by a methylating agent, TMZ, was markedly elevated in the $a d a$, ogt strain (Fig. 2). These results are in agreement with previous reports that $O^{6}$-methylguanine is efficiently repaired by AGT, which we also confirmed using a series of repair-deficient E.coli CC102 strains [20]. However, from the present results, it appears that chloroethylation might not be as efficiently repaired by AGT as methylation at the $O^{6}$ position of guanine. Previously, Mazon et al. revealed that in E. coli,

Table 3 Mutant frequencies of ACNU and BCNU induced in MMR-deficient strains

\begin{tabular}{|c|c|c|c|c|c|c|c|}
\hline & \multirow{3}{*}{$\begin{array}{l}\text { Doses } \\
(\mathrm{mM})\end{array}$} & \multicolumn{6}{|c|}{ Mutant frequency $\left(\times 10^{-7}\right)$} \\
\hline & & \multicolumn{3}{|l|}{$\mathrm{ACNU}$} & \multicolumn{3}{|l|}{$\mathrm{BCNU}$} \\
\hline & & 0 & 0.5 & 1 & 0 & 0.5 & 1 \\
\hline \multirow[t]{4}{*}{ Strain } & wild-type & $0.39 \pm 0.15$ & $22.0 \pm 5.83$ & $39.2 \pm 7.72$ & $0.59 \pm 0.62$ & $45.2 \pm 27.2$ & $118 \pm 77.3$ \\
\hline & muts & $57.2 \pm 9.11$ & $71.8 \pm 11.2$ & $90.7 \pm 9.15$ & $49.6 \pm 9.78$ & $80.8 \pm 6.43$ & $158 \pm 31.3$ \\
\hline & mutL & $15.5 \pm 2.45$ & $41.2 \pm 2.60$ & $96.4 \pm 9.26^{*}$ & $24.1 \pm 5.76$ & $100 \pm 38.2$ & $215 \pm 72.7$ \\
\hline & mutH & $34.8 \pm 7.26$ & $50.2 \pm 9.05$ & $78.9 \pm 7.72$ & $32.2 \pm 4.09$ & $107 \pm 30.0$ & $188 \pm 75.9$ \\
\hline
\end{tabular}

These data are from 3 independent experiments with ACNU and from 2 independent experiments with BCNU

Statistical analysis was performed using the Student's $t$ test. ${ }^{*} p<0.05$ compared with the mutant frequency for the wild-type 
an alkyltransferase-like protein prevents the toxicity that is induced by slightly larger $O^{6}$-alkylG adducts through the enhancement of NER and inhibition of futile MMR cycles $[31,32]$. In the present study, the lesions induced by ACNU appeared to be partly repaired by NER, because the frequency of mutations induced by ACNU in the NER-deficient ( $u v r A)$ strain increased to a similar level so that in the $a d a$, ogt strain (Fig. 2b). Numata et al. reported that some steps of NER are involved in repairing ACNU-induced DNA damage in ACNU-sensitive $\mathrm{CHO}$ cells [33]. In our results, the frequency of mutations induced by BCNU was not affected by NER deficiency at higher doses of BCNU, but it appeared to be affected at lower doses. This suggested that CENU-induced lesions might be repaired not only by AGT, but also by NER, and ACNU might cause the formation of some bulky adduct, which are mainly repaired by NER.

BCNU was mutagenic in E. coli CC104 strain only at high dose, that is, BCNU may cause a GC to TA transversion. He et al. reported the protective effects of $E$. coli formamidopyrimidine (Fapy)-DNA glycosylase and human 8-oxoguanine-DNA glycolsylase on BCNU-induced DNA damage and cell death [34]. These results show that BCNU might induce N7-chloroethylguanine in DNA followed by the formation of a Fapy residue that can induce the GC to TA transversion. N7-Chloroethylguanine might be formed more efficiently by the treatment with $\mathrm{BCNU}$ at high dose.

$O^{6}$-Chloroethylguanine rapidly transforms into unstable circularized N1- $\mathrm{O}^{6}$-ethenoguanine, then forms ICLs (reviewed in 4,6). This transformation might be responsible for the less effective repair of $O^{6}$-chloroethylguanine by AGT because $O^{6}$-chloroethylguanine should exist shortly on DNA [35]. ICLs are considered to be chemotherapeutic DNA damage, because they efficiently disrupt DNA replication such as induction of replication-mediated double strand breaks [36], leading to cell death $[5,6]$. It is well documented that recombination is involved in the repair of ICLs [5]. As such, we investigated whether recombination deficiencies affect the mutations induced by CENUs. ACNU and BCNU were toxic in recA strain of $E$. coli, and were less mutagenic than in the wild-type strain. In contrast, the mutagenicity of TMZ, which theoretically does not cause ICLs, was not affected, and no toxicity was observed at the doses tested (Fig. 3). These results suggest that the recombination of DNA is involved in the repair of ICLs, and that ICLs induce cell death if they are not repaired, as has been previously reported $[5,6]$. NER appears to be responsible for the repair of ICLs, because the incision of strands is necessary for initiating recombination. As for the mechanism of ICL repair in E. coli, the NER-recombination model is proposed [37], that is, both
NER and recombination are essential for the repair of ICLs. In this study, we constructed a new strain that was deficient in both NER and recombination to examine the involvement of NER and recombination in the toxicity and mutagenic effects of $\mathrm{ACNU}$ and $\mathrm{BCNU}$. Our results showed that the toxicity of both compounds was stronger in the double-mutant strain than in each of the single-mutant strains; however, the mutagenic effects were the similar to those in the wild-type strain and in the NER-deficient strain (Fig. 4). In other words, the toxicity appeared to be caused by the additive or synergistic effects of NER deficiency and recombination deficiency, and the mutations appeared to be mainly caused by NER deficiency. These results suggest that the ICLs induced by CENUs are independently repaired by NER and recombination. If ICLs are repaired through the NER-recombination model, the toxicity should be similar between the double-mutant strain and each single-mutant strain, because both repair systems are epistatic to each other. Previously, Berardini et al. showed another pathway for the repair of ICLs induced by nitrogen mustard, that is, the NER/DNA polymerase II pathway, which is different from the NER/recombination pathway in E. coli [38]. Recently, Cole et al. reported that an incision by NER was not required for the repair of psoralen-induced ICLs in E. coli, and that E. coli may lack an efficient repair mechanism for damage, such as ICLs [39]. At replication forks, release of ICL probably causes DNA double strand breaks inducing cytotoxicity. As the repair of double strand breaks is mediated by RecA, replication-coupled repair of ICL might perform without aid of NER. On the other hand ICLs in non-replicated regions might be repaired by NER-mediated pathways such as the translesion pathway.

It is well documented that $O^{6}$-methylguanine is efficiently repaired by both AGT (MGMT) and MMR repair systems [4]. Previously, we also revealed that methylating agents caused more mutations in AGT and MMR double-deficient strains than in each of the single-mutant strains [20]. In this study, we examined whether MMR was involved in the repair of chloroethylated lesions inducing reversion from GC to AT. As shown in Table 3, MMR does not appear to be responsible for the repair of chloroethylated lesions. There have been reports that the lesions induced by ACNU or BCNU might be repaired in a different manner from those induced by methylating agents, such as TMZ and dacarbazine, in mammalian cells $[40,41]$. These data support our present finding that MMR does not appear to have a significant role in the repair of chloroethylated lesions.

\section{Conclusions}

In conclusion, CENUs, such as ACNU and BCNU, induced GC to AT transitions in E. coli through the formation of $O^{6}$-chloroethylguanine, and consequently, 
Table 4 Summary

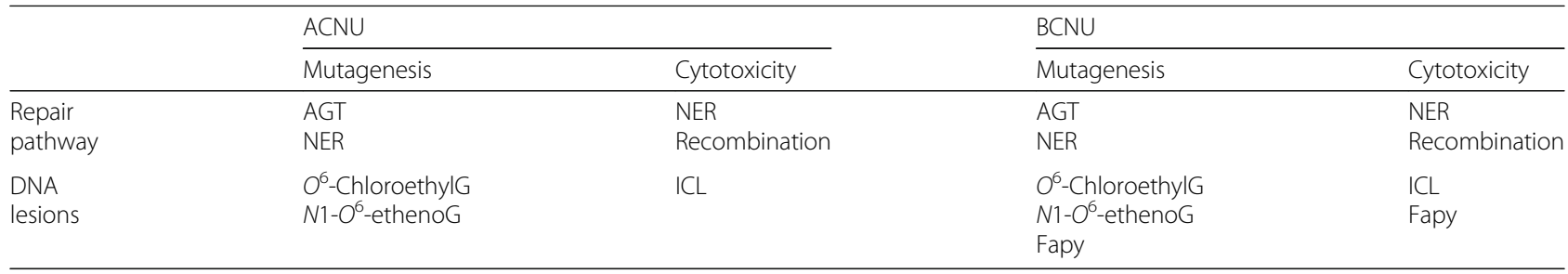

ICLs formed from the $O^{6}$-chloroethylguanine via N1- $\mathrm{O}^{6}$-ethenoguanine, causing cytotoxicity. The formation of ICLs was not mutagenic, but toxic for $E$. coli. $O^{6}$-Chloroethylguanine appears to be repaired by AGT, and perhaps also by NER, whereas ICLs appear to be independently repaired by recombination and NER. We summarize about repair pathway and potential DNA lesions in Table 4. As we used E. coli that enabled the detection of point mutations and frameshift mutations, there is a possibility that we could not observe the effects of recombination deficiencies on the mutations. In future studies, an $E$. coli strain for detecting mutations dependent on recombination should be used to examine changes in mutations due to recombination deficiencies.

\section{Abbreviations}

ACNU: Nimustine (1-[(4-amino-2-methyl-5-pyrimidinyl)methyl]-3-(2chloroethyl)-3-nitrosourea); AGT: $O^{6}$-alkylguanine-DNA alkyltransferase: BCNU: Carmustine (1,3-bis(2-chloroethyl)-1-nitrosourea);

CENU: Chloroethylnitrosourea; DMSO: Dimethylsulfoxide; ICL: Interstrand cross-link; MF: Mutant frequency; MMR: Mismatch repair; NER: Nucleotide excision repair; TMZ: Temozolomide (3,4-dihydro-3-methyl-4-oxoimidazo-[5,1d] $[1,2,3,5]$ tetrazine-8-carboxamide)

\section{Acknowledgements}

We would like to give many thanks to Dr. Toshihiro Ohta of Tokyo Pharmaceutical University for valuable suggestions in constructing the new E. coli strain for the experiment.

\section{Funding}

Not applicable.

\section{Availability of data and materials}

Not applicable.

\section{Authors' contributions}

YY and SW carried out all experiments and performed the data analysis. KO and SA participated in the design of the study and discussion with the results. ET and KN participated in the construction of new E. coli strain and discussion with the results. TN conceived of the study, participated in its design and coordination, and described the manuscript. All authors read and approved the final manuscript.

Ethics approval and consent to participate

Not applicable.

\section{Consent for publication}

Not applicable.

\section{Competing interests}

The authors declare that they have no competing interests.

\section{Publisher's Note}

Springer Nature remains neutral with regard to jurisdictional claims in published maps and institutional affiliations.

\section{Author details \\ 'Graduate School of Medicine, Dentistry, and Pharmaceutical Sciences, Okayama University, Tsushima-naka, Kita-ku, Okayama 700-8530, Japan. ${ }^{2}$ Faculty of Pharmaceutical Sciences, Okayama University, Tsushima-naka, Kita-ku, Okayama 700-8530, Japan. ${ }^{3}$ Nihon Pharmaceutical University, Ina, Kita-Adachi-Gun, Saitama 362-0806, Japan. ${ }^{4}$ Present address: Collaborative Research Center of Okayama University for Infectious Diseases in India, National Institute of Cholera and Enteric Diseases JICA Building ID Hospital Campus, Beliaghata Kolkata 700010, India.}

Received: 21 November 2018 Accepted: 4 March 2019

Published online: 05 April 2019

\section{References}

1. Hurley LH. DNA and associated processes as targets for cancer therapy. Nat Rev Cancer. 2002;2:188-200.

2. Middleton MR, Margison GP. Improvement of chemotherapy efficacy by inactivation of a DNA-repair pathway. Lancet Oncol. 2003;4:37-44.

3. Fu D, Calvo JA, Samson LD. Balancing repair and tolerance of DNA damage caused by alkylating agents. Nat Rev Cancer. 2012;12:104-20.

4. Drabløs F, Feyzi E, Aas PA, Vaagbø CB, Kavli B, Bratlie MS, Peña-Diaz J, Otterlei M, Slupphaug G, Krokan HE. Alkylation damage in DNA and RNA-repair mechanisms and medical significance. DNA Repair (Amst). 2004;3:1389-407.

5. Nikolova T, Hennekes F, Bhatti A, Kaina B. Chloroethylnitrosourea-induced cell death and genotoxicity: cell cycle dependence and the role of DNA double-strand breaks. HR and NHEJ Cell Cycle. 2012;11:2606-19.

6. Nikolova T, Roos WP, Krämer OH, Strik HM, Kaina B. Chloroethylating nitrosoureas in cancer therapy: DNA damage, repair and cell death signaling. Biochim Biophys Acta. 2017;1868:29-39.

7. Wang Z, Sun H, Yakisich JS. Overcoming the blood-brain barrier for chemotherapy: limitations, challenges and rising problems. Anti Cancer Agents Med Chem. 2014:14:1085-93.

8. Liu HL, Hua MY, Chen PY, Chu PC, Pan CH, Yang HW, Huang CY, Wang JJ, Yen TC, Wei KC. Blood-brain barrier disruption with focused ultrasound enhances delivery of chemotherapeutic drugs for glioblastoma treatment. Radiology. 2010;255:415-25.

9. Swann PF. Why do $O^{6}$-alkylguanine and $O^{4}$-alkylthymine miscode? The relationship between the structure of DNA containing $O^{6}$-alkylguanine and $\mathrm{O}^{4}$-alkylthymine and the mutagenic properties of these bases. Mutat Res. 1990;233:81-94.

10. Tong WP, Kirk MC, Ludlum DB. Formation of the cross-link 1- $\left[N^{3}\right.$ deoxycytidyl], 2-[N'-deoxyguanosinyl]ethane in DNA treated with $N, N^{\prime}$-bis(2-chloroethyl)-N-nitrosourea. Cancer Res. 1982;42:3012-5.

11. Dronkert ML, Kanaar R. Repair of DNA interstrand cross-links. Mutat Res. 2001:486:217-47.

12. Ludlum DB. DNA alkylation by the haloethynitrosoureas: nature of modifications produced and their enzymatic repair or removal. Mutat Res. 1990;233:117-26.

13. Li L, Li S, Sun G, Peng R, Zhao L, Zhong R. Influence of the expression level of $O^{6}$-alkylguanine-DNA alkyltransferase on the formation of DNA interstrand crosslinks induced by chloroethylnitrosoureas in cells: a quantitation using high-performance liquid chromatography-mass spectrometry. PLoS One. 2015;10:e0121225.

14. Preuss I, Thust R, Kaina B. Protective effect of $O^{6}$-methylguanine-DNA methyltransferase (MGMT) on the cytotoxic and recombinogenic activity of different antineoplastic drugs. Int J Cancer. 1996;65:506-12. 
15. Verbeek B, Southgate TD, Gilham DE, Margison GP. $O^{6}$-Methylguanine-DNA methyltransferase inactivation and chemotherapy. Br Med Bull. 2008;85:17-33.

16. Wiencke JK, Wiemels J. Genotoxicity of 1,3-bis(2-chloroethyl)-1-nitrosourea (BCNU). Mutat Res. 1995:339:91-119.

17. Cupples CG, Miller JH. A set of lacZ mutations in Escherichia coli that allow rapid detection of each of the six base substitutions. Proc Natl Acad Sci U S A. 1989;86:5345-9.

18. Cupples CG, Cabrera M, Cruz C, Miller JH. A set of lacZ mutations in Escherichia coli that allow rapid detection of specific frameshift mutations. Genetics. 1990;125:275-80.

19. Negishi K, Loakes D, Schaaper RM. Saturation of DNA mismatch repair and error catastrophe by a base analogue in Escherichia coli. Genetics. 2002;161: 1363-71.

20. Taira K, Kaneto S, Nakano K, Watanabe S, Takahashi E, Arimoto S, Okamoto K, Schaaper RM, Negishi K, Negishi T. Distinct pathways for repairing mutagenic lesions induced by methylating and ethylating agents. Mutagenesis. 2013;28:341-50

21. Watanabe-Akanuma M, Ohta T. Effects of DNA repair deficiency on the mutational specificity in the lacZ gene of Escherichia coli. Mutat Res. 1994; 311:295-304.

22. Takahashi E, Okamoto K, Arimoto S, Yamanaka H, Negishi T. Involvement of the drug efflux protein TolC in mutagenicity induced by MNNG or Trp-P-2. Mutat Res. 2006:605:42-50.

23. Taira K, Nakamura S, Nakano K, Maehara D, Okamoto K, Arimoto S, Loakes D, Worth L Jr, Schaaper RM, Seio K, Sekine M, Negihsi K, Negishi T. Binding of MutS protein to oligonucleotides containing a methylated or an ethylated guanine residue, and correlation with mutation frequency. Mutat Res. 2008;640:107-12.

24. Nakano K, Yamada Y, Takahashi E, Arimoto S, Okamoto K, Negishi K, Negishi T. E. coli mismatch repair enhances AT-to-GC mutagenesiss caused by alkylating agents. Mutat Res. 2017;815:22-7.

25. Rasmussen LJ, Samson L. The Escherichia coli MutS DNA mismatch binding protein specifically binds $0^{6}$-methylguanine DNA lesions. Carcinogenesis. 1996;17:2085-8.

26. Stojic $L$, Brun $R$, Jiricny J. Mismatch repair and DNA damage signalling. DNA Repair (Amst). 2004;3:1091-101.

27. Tashima M, Sawada H, Uchino H, Nishioka H. Effect of 1,3-bis (2 chloroehtyl)-29.-nitrosourea on Escherichia coli DNA repair system. Gan. 1978;69:695-8

28. Sato K, Kitajima Y, Koga Y, Miyazaki K. The effect of $O^{6}$-methylguanine-DNA methyltransferase (MGMT) and mismatch repair gene (hMLH1) status on the sensitivity to alkylating agent 1-(4-amino-2-methyl-5-pyrimidinyl) methyl-3(2-chloroethyl)-3-nitrosourea (ACNU) in gallbladder carcinoma cells. Anticancer Res. 2005;25:4021-8.

29. Suzuki M, Sugimoto $Y$, Tsuruo T. Efficient protection of cells from the genotoxicity of nitrosoureas by the retrovirus-mediated transfer of human $0^{6}$-methylguanine-DNA methyltransferase using bicistronic vectors with human multidrug resistance gene 1. Mutat Res. 1998:401:133-41.

30. Becker K, Gregel CM, Kaina B. The DNA repair protein $0^{6}$-methylguanineDNA methyltransferase protects against skin tumor formation induced by antineoplastic chloroethylnitrosourea. Cancer Res. 1997;57:3335-8.

31. Mazon G, Philippin G, Cadet J, Gasparutto D, Fuchs RP. The alkyltransferaselike ybaZ gene product enhances nucleotide excision repair of $0^{6}$ alkylguanine adducts in E. coli. DNA Repair (Amst). 2009;8:697-703.

32. Mazon G, Philippin G, Cadet J, Gasparutto D, Modesti M, Fuchs RP. Alkyltransferase-like protein (eATL) prevents mismatch repair-mediated toxicity induced by $\mathrm{O}^{6}$-alkylguanine adducts in Escherichia coli. Proc Natl Acad Sci U S A. 2010;107:18050-5.

33. Numata $M$, Hata $H$, Tohda $H$, Yasui $A$, Oikawa A. DNA repair pathways in mammalian cells analyzed by isolation of ACNU-sensitive Chinese hamster ovary cells. Tohoku J Exp Med. 1992;168:123-8.

34. He YH, Xu Y, Kobune M, Wu M, Kelley MR, Martin WJ 2nd. Escherichia coli FPG and human OGG1 reduce DNA damage and cytotoxicity by BCNU in human lung cells. Am J Physiol Lung Cell Mol Physiol. 2002;282:L50-5.

35. Pegg AE, Kanugula S, Loktionova NA. $O^{6}$-Alkylguanine-DNA alkyltransferase. In: Penning MT, editor. Chemical Carcinogenesis. Totowa: Humana Press; 2011. p. 321-43.

36. Bessho T. Induction of DNA replication-mediated double strand breaks by psoralen DNA interstrand cross-links. J Biol Chem. 2003;278:5250-4.

37. Sladek FM, Munn MM, Rupp WD, Howard-Flanders P. In vitro repair of psoralen-DNA cross-links by RecA, UVrABC, and the $5^{\prime}$-exonuclease of DNA polymerase I. J Biol Chem. 1989;264:6755-65.
38. Berardini M, Foster PL, Loechler EL. DNA polymerase II (polB) is involved in a new DNA repair pathway for DNA intastrand cross-links in Escherichia coli. J Bacteriol. 1999;181:2878-82.

39. Cole JM, Acott JD, Courcelle CT, Courcelle J. Limited capacity or involvement of excise on repair, double strand breaks, or translesion synthesis for psoralen cross-link repair in Eschericha coli. Genetics. 2018;210:99-112.

40. Pepponi R, Marra G, Fggetta MP, Falcinelli S, Pagani E, Bonmassar E, Jiricny J, $D^{\prime}$ Atri $S$. The effect of $O^{6}$-alkylguanine-DNA alkyltransferase and mismatch repair activities on the sensitivity of human melanoma cells to temozolomide, 1,3-bis(2-chloroethyl)1-nitrosourea, and cisplatin. J Pharacol Exp Ther. 2003;304:661-8.

41. Sanada M, Hidaka M, Takagi Y, Takano TY, Nakatsu Y, Tsuzuki T, Sekiguchi M. Modes of actions of two types of anti-neoplastic drugs, dacarbazine and ACNU, to induce apoptosis. Carcinogenesis. 2007;28:2657-26.

\section{Ready to submit your research? Choose BMC and benefit from:}

- fast, convenient online submission

- thorough peer review by experienced researchers in your field

- rapid publication on acceptance

- support for research data, including large and complex data types

- gold Open Access which fosters wider collaboration and increased citations

- maximum visibility for your research: over $100 \mathrm{M}$ website views per year

At BMC, research is always in progress.

Learn more biomedcentral.com/submissions 